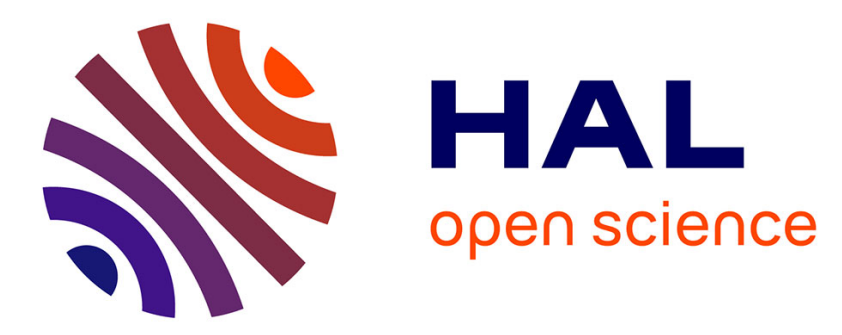

\title{
Relationship between the elastic properties and the order parameter of the spin-density wave phase of (TMTSF)2PF6
}

\author{
S. Brown, B. Alavi, W. Clark, M. Hanson, B. Klemme
}

\section{To cite this version:}

S. Brown, B. Alavi, W. Clark, M. Hanson, B. Klemme. Relationship between the elastic properties and the order parameter of the spin-density wave phase of (TMTSF)2PF6. Journal de Physique IV Proceedings, 1993, 03 (C2), pp.C2-225-C2-230. 10.1051/jp4:1993245 . jpa-00251328

\section{HAL Id: jpa-00251328 https://hal.science/jpa-00251328}

Submitted on 1 Jan 1993

HAL is a multi-disciplinary open access archive for the deposit and dissemination of scientific research documents, whether they are published or not. The documents may come from teaching and research institutions in France or abroad, or from public or private research centers.
L'archive ouverte pluridisciplinaire HAL, est destinée au dépôt et à la diffusion de documents scientifiques de niveau recherche, publiés ou non, émanant des établissements d'enseignement et de recherche français ou étrangers, des laboratoires publics ou privés. 


\title{
Relationship between the elastic properties and the order parameter of the spin-density wave phase of (TMTSF) ${ }_{2} \mathrm{PF}_{6}$
}

\author{
S.E. BROWN, B. ALAVI, W.G. CLARK, M.E. HANSON and B. KLEMME \\ Department of Physics, UCLA, Los Angeles, CA 900024, U.S.A.
}

\begin{abstract}
We present elastic constant data of the material (TMTSF) ${ }_{2} \mathrm{PF}_{6}$, with particular emphasis on the region about the phase transition, and at low temperatures. Specific heat, resistivity, and NMR measurements have indicated a very sharp transition at $\mathrm{T}_{\mathrm{SDW}}=12.1 \mathrm{~K}$. Similarly sharp features have been observed in the temperature dependence of the Young's Modulus, followed by a continual stiffening below the transition. Comparison to recent NMR results indicate that the modulus varies as the square of the internal magnetic field below $\mathrm{T}_{\mathrm{SDW}}$, thus constraining the form of the coupling of the order parameter to the strain field. There is no evidence for anomalies in the modulus below $4 \mathrm{~K}$, where it has been suggested that a transition to a commensurate state occurs.
\end{abstract}

The onset of spin-density wave order in the organic crystal (TMTSF) ${ }_{2} \mathrm{PF}_{6}$ is markedly different from the Peierls transition in linear chain compounds such as $\mathrm{TaS}_{3}, \mathrm{~K}_{0.3} \mathrm{MoO}_{3}$, and $\left(\mathrm{TaSe}_{4}\right)_{2} \mathrm{I}$. Transport measurements indicate a particularly sharp onset of the insulating phase, and probes of the internal field suggest that the transition is discontinuous [1,2,3]. Questions regarding the nature of the phase transition and the collective effects motivated us to re-examine the behavior of the Young's Modulus in the spin-density wave phase.

Many charge-density wave materials show a stiffening of elastic moduli below the phase transition, as well as a softening when the depinning electric field is exceeded. Quantitative understanding of these effects has proven to be difficult for a number of reasons. For example, the high temperatures involved and large fluctuation effects lead to uncertainties in the background behavior, making it difficult to evaluate the effects of CDW formation and depinning. In contrast, for $(\mathrm{TMTSF})_{2} \mathrm{PF}_{6}$, the transition is sharp, and deviations are well-defined. Below we present data on the Young's Modulus and make direct comparisons to the order parameter.

The sound velocity $v$ associated with the Young's modulus was measured in a standard vibrating-reed configuration, where the sample was driven electrostatically in the lowest order bending 
mode resonance, and the relative amplitude of the motion was detected by capacitive coupling of the sample to another electrode. In the range of temperatures overlapping the transition, the overall behavior is similar to previous results $[4,5,6]$.

Figure 1 shows the temperature dependence of the change in the bending mode sound velocity for a sample from the same preparation as that used for the proton NMR measurements described in Ref. [1]. With the external field applied in the $b^{\prime}$ direction, a broadening of the linewidth results from the development of a static internal field $\mathrm{H}_{\mathrm{int}}$ at the proton sites associated with SDW formation. In order to compare the elastic measurements with those of the internal field, a background needs to be subtracted. Although we cannot say how the background elasticity should should change with temperature below $\mathrm{T}_{\mathrm{SDW}}$, the choice should not be crucial close to $12 \mathrm{~K}$ where the modulus is changing rapidly. For the NMR measurements, we subtract the dipolar contribution of the metallic phase. The plot in Fig. 2 uses the the solid line of Fig. 1 as the background. Two samples are shown to exhibit a change in the modulus that follows very closely to the square of the internal field as reported in Ref. [1].

The functional similarity of $\mathrm{H}_{\mathrm{int}}$ to $\delta \mathrm{v} / \mathrm{v}$ can be understood using a phenomenological Landau potential that includes coupling terms to the strain field. In principle, the low symmetry of the crystal could lead to a large number of allowable terms. Fortunately, we have found that the simplest possible model giving a first order transition describes the NMR linewidth very well, namely, $H_{i n t}(T)$ is welldescribed by an expansion terminated at the $6^{\text {th }}$ order, and using a negative fourth order term [7]. Continuing along similar lines, we forego the full tensor description and instead speak of the general consequences of the form of the coupling. For instance, consider a Landau coupling term of the form

$F^{t}=\eta e^{2} H_{i n t}{ }^{2}+(1 / 2) c_{0} e^{2}$

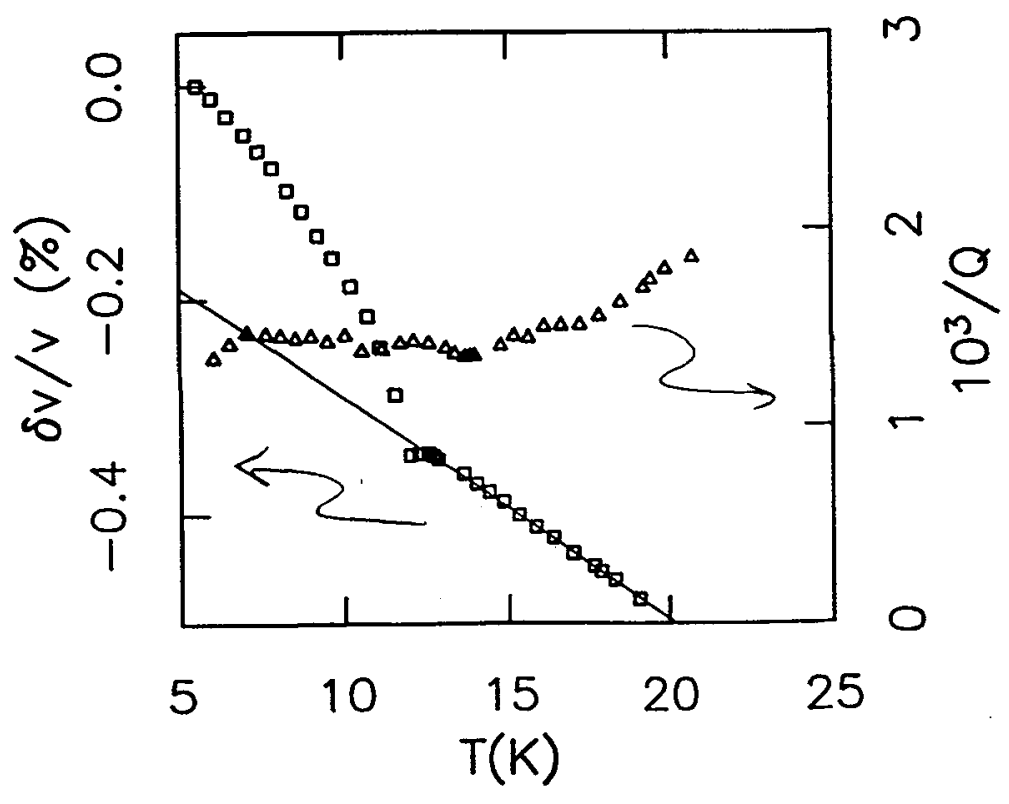

Fig. 1. Temperature

dependences of the relative change in sound velocity ( ), and internal friction in the vicinity of $\mathbf{T}_{\text {SDW. }}$. The full line is a fit used in Fig. 2. 
with $\eta$ a constant, $c_{0}$ the elastic constant above the transition, and e the strain. For this simple model, $\delta \mathrm{v} / \mathrm{v} \sim\left(\eta / \mathrm{c}_{0}\right) \mathrm{H}_{\text {int }}{ }^{2}$ below the transition.

In some cases, strain-coupling to the order parameter leads to spontaneous strain and a stressdependent transition temperature. Indeed, $\mathrm{T}_{\mathrm{SDW}}$ drops very quickly with applied pressure [8]. However, the coupling term in Eq. (1) does not lead to this characteristic, and thus this feature must arise from a different coupling term. The significance is that it is not necessary (from the experiments that we know about) to insist that this contribution to the elastic constant be intrinsic.

Maki and Virosztek [9] have proposed a model for $\delta \mathrm{v} / \mathrm{v}$ in density-wave systems in which the stiffening below the transition occurs in conjunction with the pinning of the DW. Coupling to the electronic system in the normal state occurs in the usual way, where the lattice is softened as a result of the dielectric response of the electrons to the ionic strain. The ability of the conduction electrons to

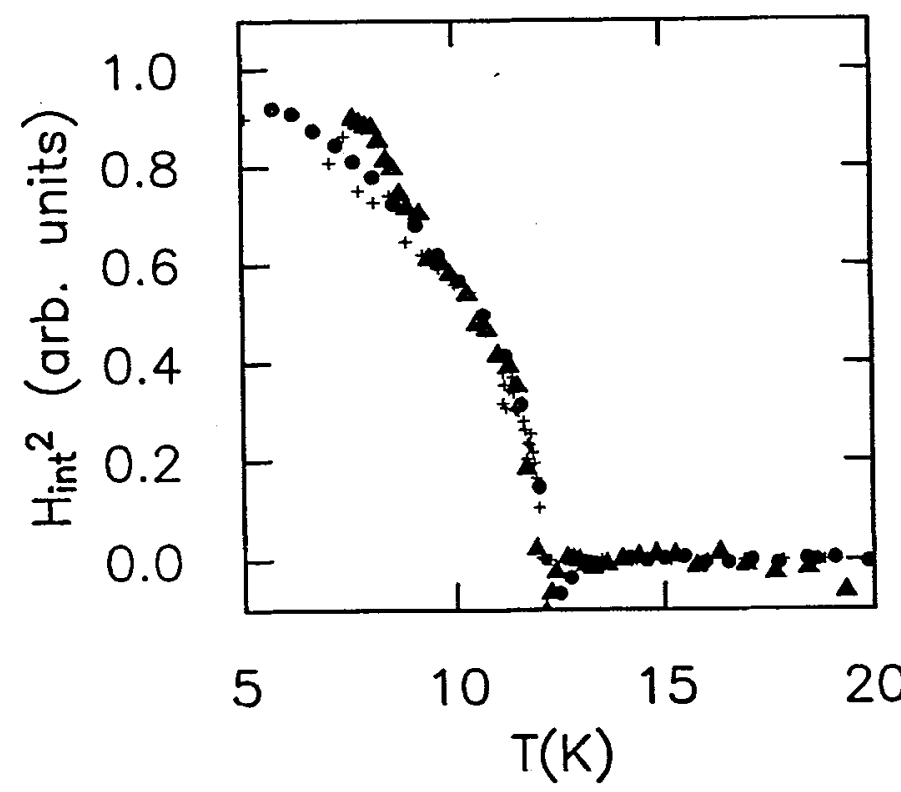

screen the Coulomb respulsion in the SDW phase is reduced when the density wave is pinned. The form for the temperature dependence of the sound velocity near to $\mathrm{T}_{\mathrm{SDW}}$ is

$\frac{\delta \mathrm{v}}{\mathrm{v}}=\frac{\rho_{\mathrm{s}}}{\rho}$,

with $\rho_{s}$ the superfluid density. Note that the appearance of the superfluid density in Eq. [2] represents the quasiparticle response rather than a stiffness directly associated with the SDW. With $\rho_{\mathrm{s}} \sim \Delta^{2}$, and the usual linear variation of the single-particle energy gap to $\mathrm{H}_{\text {int }}$, Eq. (2) suggests that allowing for the quasiparticle response gives the correct functional form for the data. A potential problem with this approach is the sample-sample variation in the overall change reported by different authors. Experiments by Shi, et al [3], Brill, et al [4], and previous results by us [5] indicate a variation for the sound velocity change to range from $0.5 \%-2 \%$. However, this could also arise from experimental problems, including 
macroscopic imperfections that lead to impure resonant modes or microscopic inhomogeneities that lead to relaxational processes of some kind.

One way for the strain to couple directly to the order parameter is through the imperfect nesting parameter. The strong reduction in the critical temperature with applied pressure is generally attributed to imperfect nesting. In a phenomenological Landau theory, this aspect is accounted for by including intrinsic coupling terms of the form $\eta^{\prime} \mathrm{eH}_{\mathrm{int}}{ }^{2}$. In addition to $\mathrm{T}_{\mathrm{c}}(\mathrm{P})$, a spontaneous strain will develop in the low-temperature phase. In the simple Landau model described here, the resulting temperature dependence of the elastic constant does not fit the data.

Mozurkewich [10] has pointed out that pinning will prevent the system from reaching a free energy minimum. The net cost would have to be an even function of $\mathrm{e}$, so to lowest order in the strain, this affect can be included phenomenologically in the form of Eq. [1]. As before, it is not an intrinsic contribution. However, unlike the discussion in Ref. [9], the importance varies as $\rho_{\mathrm{s}}$ because of coupling to the degrees of freedom of the spin-density wave through the impurity pinning. Another major difference is that in the calculation of Maki and Virosztek, the time scales of the effects are such that there should be no frequency-dependent effects. This restriction is relaxed in the model of Ref. [10].

Finally, we examine the behavior at low temperatures. The motivation is twofold. First, measurements down to $4 \mathrm{~K}$ indicate that stiffening continues to lower temperatures, and generally dv/dT vanishes at $\mathrm{T}=0$ for crystalline materials. Second, there are many experiments, including NMR longitudinal relaxation [11], transport [12] and specific heat [13], that indicate a change in the state or dynamics of the SDW below approximately $3.5 \mathrm{~K}$. There has been some speculation that the system undergoes a commensurability transition. In charge-density wave systems, such as the dichalcogenides, the elastic modulus is strongly affected if there is a well-defined lock-in transition [14]. Figure 3 shows

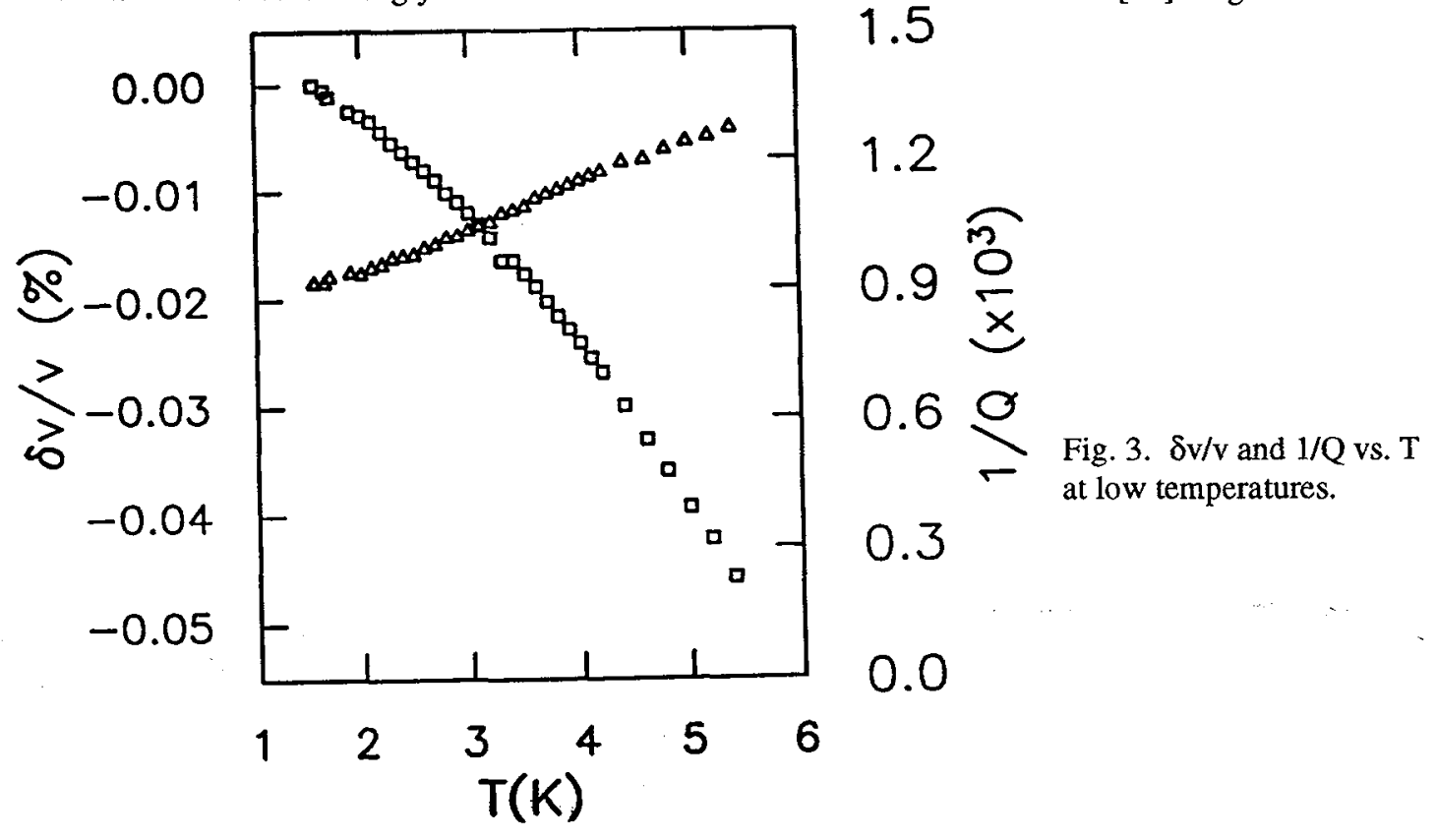


our low-temperature data for the Young's Modulus and the internal friction. At most, there is a weak effect in the internal friction, and nothing discernible in the modulus. At the same time, the quality factor is high enough to enable a determination of the resonant frequency to 1-2 ppm.

The null result in the neighborhood of $3.5 \mathrm{~K}$ could indicate that whatever is causing anomlies in other physical measurements does not couple to the Young's Modulus. In contrast, the elastic properties of the layered dichalcogenides (such as $2 \mathrm{H}-\mathrm{TaSe}_{2}$ ) change drastically at the first-order incommensurate/commensurate transition. A contributing factor is domain formation that results from the existence of a triply degenerate q-vector in the dichalcogenides. We know of no reason to require the same kind of domains for the SDW phase of $(\mathrm{TMTSF})_{2} \mathrm{PF}_{6}$. Nevertheless, it is not immediately evident that pinning of the incommensurate phase, should it occur, should play the same role in the elastic modulus below $3.5 \mathrm{~K}$ if it is a lock-in transition. Another possibility is that the low temperature is due to a crossover from 2D to 3D spin wave correlations [15]. We are unsure how this might effect the sound velocities. Finally, An alternative interpretation of the NMR measurements in terms of fluctuation dynamics without a phase transition has been suggested. The lack of an associated anomaly in the elasticity could then be a question of time-scale of the probe. Experiments are underway exploring this possibility.

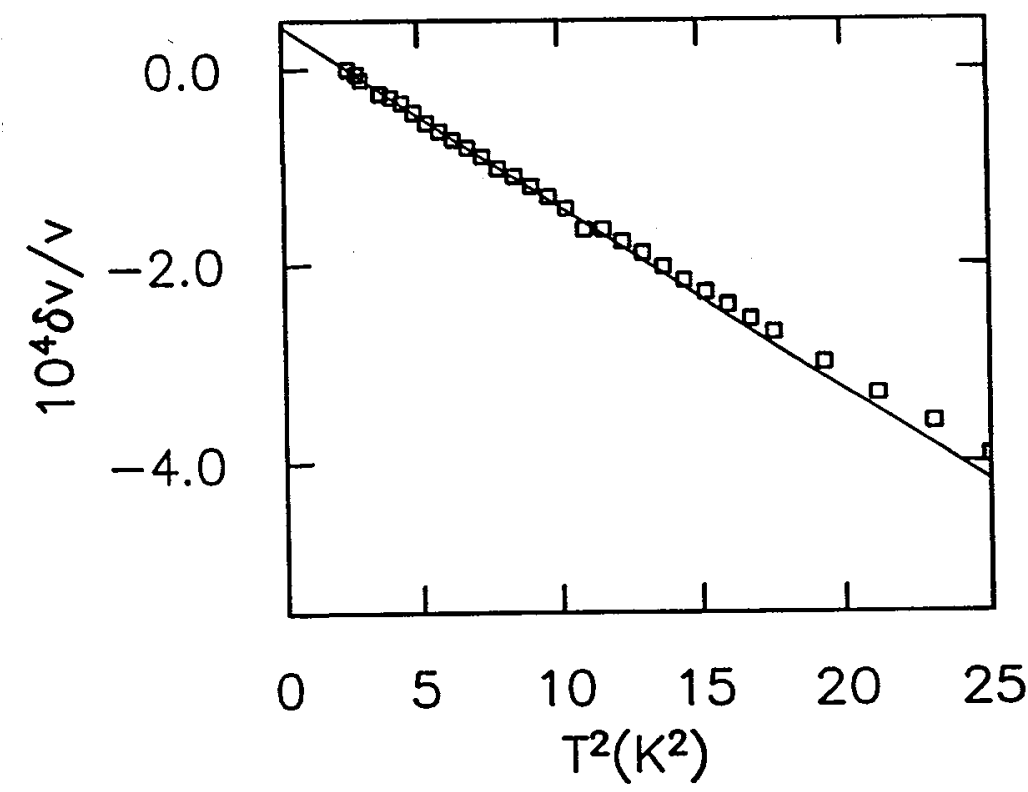

Fig. 4. Relative change in sound velocity vs. $\mathrm{T}^{2}$.

The change in sound velocity from $1.5 \mathrm{~K}-4 \mathrm{~K}$ as a function of $\mathrm{T}^{2}$ is shown in Fig. 4. We only want to point out that this is unusual for an insulating crystalline solid. It can occur in metals because of the shift in the Fermi energy at finite temperatures. Insulators are usually described by a higher power law in $\mathrm{T}$, or exponentially as $1 / \mathrm{T}[16]$. Whether the magnetism is playing a role here, we are unsure, but the observed changes are somewhat larger than a large array of materials. 
In summary, we have shown that the sound velocity in (TMTSF) ${ }_{2} \mathrm{PF}_{6}$ varies as the square of the internal magnetic field. The form of the strain-coupling to the order parameter is probably not intrinsic, and we have discussed the possible connection of imperfect nesting to the observations. At low temperatures, we see no evidence for an anomaly between $3-4 \mathrm{~K}$. The details of why this should be, if it is a lock-in transition,remain unclear. Finally, the modulus varies as $\mathrm{T}^{2}$ at low temperatures.

ACKNOWLEDGEMENTS: We would like to thank T. Csiba, S. Donovan, G. Gruner, and K. Maki for discussions. This work was supported in part by the National Science Foundation under grant no. DMR9296085.

\section{References}

1. W.G. Clark, M.E. Hanson, W.H. Wong, and B. Alavi (Proceedings of LT-20, Physica B+C, to be published).

2. L.P. Le, et al., Europhys. Lett. 15, 547 (1991).

3. T. Takahashi, et al., Physica 143B, 417 (1986).

4. P.M. Chaikin, et al., Solid State Commun. 41, 739 (1982), X.D. Shi, et al., Mat. Res. Soc. Symp. 173, 239 (1990).

5. J.W. Brill (private communication).

6. S.E. Brown, et al., Phys. Rev. B 46, 10483 (1992).

7. S.E. Brown, et al. (to be published).

8. See, for example, D. Jerome and H.J. Schulz, Adv. Phys. 31, 299 (1982).

9. K. Maki and A. Virosztek, Phys. Rev. B 36, 2910 (1987); A. Virosztek and K. Maki, Mat. Res. Soc. Symp. Proc., 173, 233 (1990).

10. G. Mozurkewich, Phys. Rev. B 42, 11183 (1990).

11. See, for example, W.H. Wong, et al., Phys. Rev. Lett. 70, 1882 (1993), and references therein.

12. See, for example, S. Tomic, et al., J. de Physique I, 1, 1603 (1991); G. Mihaly, et al., Phys. Rev. Lett. 67, 2713 (1991).

13. J.C. Lasjaunias, et al., Solid State Commun. 84, 297 (1992).

14. M. Barmatz, et al., Phys. Rev. B 12, 4367 (1975).

15. T. Csiba (private communication).

16. Y.P. Varshni, Phys. Rev. B 2, 3952 (1970). 